\title{
High Flux Isotope Reactor Quarterly Report January, February, and March of 1977
}
B. L. Corbett
R. V. McCord
K. H. Poteet

\section{OAK RIDGE NATIONAL LABORATORY}




\section{DISCLAIMER}

This report was prepared as an account of work sponsored by an agency of the United States Government. Neither the United States Government nor any agency Thereof, nor any of their employees, makes any warranty, express or implied, or assumes any legal liability or responsibility for the accuracy, completeness, or usefulness of any information, apparatus, product, or process disclosed, or represents that its use would not infringe privately owned rights. Reference herein to any specific commercial product, process, or service by trade name, trademark, manufacturer, or otherwise does not necessarily constitute or imply its endorsement, recommendation, or favoring by the United States Government or any agency thereof. The views and opinions of authors expressed herein do not necessarily state or reflect those of the United States Government or any agency thereof. 


\section{DISCLAIMER}

Portions of this document may be illegible in electronic image products. Images are produced from the best available original document. 


\section{Printed in the United States of America. Available from National Technical Information Service \\ U.S. Department of Commerce 5285 Port Royal Road, Springfield, Virginia 22161 \\ Price: Printed Copy $\$ 4.00$; Microfiche $\$ 3.00$}

This report was prepared as an account of work sponsored by the United States Government. Neither the United States nor the Energy Research and Development Administration/United States Nuclear Regulatory Commission, nor any of their employees, nor any of their contractors, subcontractors, or their employees, makes any warranty, express or implied, or assumes any legal liability or responsibility for the accuracy, completeness or usefulness of any information, apparatus, product or process disclosed, or represents that its use would not infringe privately owned rights. 
Contract No. W-7405-eng-26

Operations Division

HIGH FLUX ISOTOPE REACTOR QUARTERLY REPORT

JANUARY, FEBRUARY, AND MARCH OF 1977

B. L. Corbett

R. V. McCord

K. H. Poteet

Date Published - June 1977

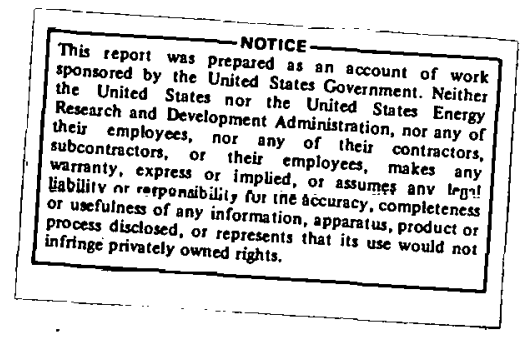

NOTICE This document contains information of a preliminary nature. It is subject to revision or correction and therefore does not represent a final report.

\author{
OAK RIDGE NATIONAL LABORATORY \\ Oak Ridge, Tennessee 37830 \\ operated by \\ UNION CARBIDE CORPORATION \\ for the \\ ENERGY RESEARCH AND DEVELOPMENT ADMINISTRATION
}

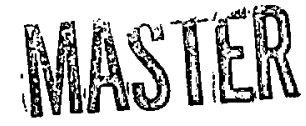




\section{THIS PAGE}

\section{WAS INTENTIONALLY \\ LEFT BLANK}


CONTENTS

SUMMARY . . . . . . . . . . . . . . . . . . . 1

OPERATIONS . . . . . . . . . . . . . . . . . . . . 1

SHUTDOWNS . . . . . . . . . . . . . . . . . . . . . 2

Plant MatnTEnANCE . . . . . . . . . . . . . . . . . . . . 4

INSTRUMENTATION AND CONTROLS . . . . . . . . . . . . . . . 4

SYSTEM SURVEILLANCE TESTS AND RESULTS . . . . . . . . . . . 6

Vessel Head Studs . . . . . . . . . . . . . . 6

Semiannual Reactor-Component Inspection . . . . . . . . 6

Stack Filters....................... . 7

REACTOR DESIGN CHANGES .................. . . . 9

REVISIONS TO THE HFIR OPERATING MANUAL . . . . . . . . . . . 10

REACTOR EXPERTMENTS . . . . . . . . . . . . . . . . . . 10

Experimental Facilities . . . . . . . . . . . . 10

HFIR Target Loading . . . . . . . . . . . . . . . . . II

DISTRIBUTION . . . . . . . . . . . . . . . . . 17 


\section{SUMMARY}

Routine reactor operation with four end-of-cycle shutdowns this quarter resulted in an on-stream time of $94.5 \%$. The semiannual reactorcomponent inspection was completed, and the control plates were changed.

\section{OPERATIONS}

Basic operating data for the quarter are listed in Table 1.

Table 1. HFIR Basic Operating Data

(January 1, 1977 through March 31, 1977)

\begin{tabular}{|c|c|c|c|}
\hline & $\begin{array}{c}\text { This } \\
\text { Quarter }\end{array}$ & $\begin{array}{c}\text { Last } \\
\text { Quarter }\end{array}$ & $\begin{array}{l}\text { Year } \\
\text { To Date }\end{array}$ \\
\hline Total energy, MWd & 8,455 & 8,323 & 8,455 \\
\hline Average power, MW/operating hr & 99.4 & 99.0 & 99.4 \\
\hline Time operating, \% & 94.5 & 96.5 & 94.5 \\
\hline Reactor availability, \% & 95.1 & 96.8 & 95.1 \\
\hline $\begin{array}{l}\text { Reactor water radioactivity, } \\
\frac{\text { c/min }}{\mathrm{ml}} \text { (av) }\end{array}$ & 356,000 & 313,000 & \\
\hline $\begin{array}{l}\text { Pool water radioactivity, } \\
\frac{\mathrm{c} / \mathrm{min}}{\mathrm{m} 1} \text { (av) }\end{array}$ & 49 & 52 & \\
\hline
\end{tabular}

The starting and ending dates for cycles 141, 142, 143, and 144 are presented in Table 2. Cycle 145 was still in progress at the end of the quarter and $404 \mathrm{MWd}$ had been accumulated.

Table 2. Cycles of Operation

\begin{tabular}{clll}
\hline Cycle No. & Date Started & Date Ended & $\begin{array}{c}\text { Accumulated } \\
\text { Power (MWd) }\end{array}$ \\
\hline 141 & $12-15-76$ & $1-10-77$ & 2368 \\
142 & $1-10-77$ & $2-3-77$ & 2398 \\
143 & $2-5-77$ & $3-1-77$ & 2448 \\
144 & $3-2-77$ & $3-26-77$ & 2345 \\
1.45 & $3-27-77$ & In progress & 404 \\
\hline
\end{tabular}


The status of the HFIR fuel and control-plate inventory is indicated in Table 3.

Table 3. HFIR Material Inventory

\begin{tabular}{lcc}
\hline \multicolumn{1}{c}{ Item } & This & Quart \\
Quarter & Quarter \\
New fuel elements placed in service & 4 & 3 \\
New fuel elements available for use & 45 & 49 \\
Spent fuel elements on hand & 13 & 9 \\
Spent fuel elements shipped & 1 & 7 \\
New sets of shim plates placed in service & 0 & 0 \\
New sets of shim plates available for use & 2 & 2 \\
\hline
\end{tabular}

\section{SIIUTDOWNS}

A summary of the HFIR shutdowns for the quarter is presented in Table 4. Table 5 gives further details of the shutdowns.

Table 4. Summary of HFIR Shutdowns

\begin{tabular}{|c|c|c|}
\hline Type & Number & Downtime (hr) \\
\hline \multicolumn{3}{|c|}{ Scheduled } \\
\hline End of syolc & 4 & 118.784 \\
\hline \multicolumn{3}{|c|}{ Unscheduled } \\
\hline Controller error & 1 & 0.133 \\
\hline
\end{tabular}


Table 5. Description of HFIR Shutdowns

\begin{tabular}{|c|c|c|}
\hline Date & $\begin{array}{l}\text { Downtime } \\
(\mathrm{hr})\end{array}$ & Remarks \\
\hline \multicolumn{3}{|r|}{ Scheduled } \\
\hline $1-10-77$ & 18.917 & $\begin{array}{l}\text { Fuel cycle } 141 \text { was completed at } 1: 15 \text { a.m. A total power generation of } 2368 \text { MWd } \\
\text { was obtained on fuel elements } 138-0 \text { and } 138-\mathrm{I} \text {. The shutdown was extended } \\
\sim 4 \text { hours to complete experiment removal. }\end{array}$ \\
\hline $2-3-77$ & 34.5 & $\begin{array}{l}\text { Fuel cycle } 142 \text { was completed at } 8: 10 \mathrm{p} . \mathrm{m} \text {. A total power generation of } 2398 \text { MWd } \\
\text { was obtained on fuel elements } 139-0 \text { and } 139-\mathrm{I} \text {. Outer control plate } 9-3 \text {, which } \\
\text { was fitted with test journal bearings, was removed from position No. 3. Plate } \\
10-3 \text {, also fitted with test journal bearings, was installed in its place. }\end{array}$ \\
\hline $3-1-77$ & 22.95 & $\begin{array}{l}\text { Fuel cycle } 143 \text { was completed at } 6: 09 \mathrm{p} . \mathrm{m} \text {. A total power generation of } 2448 \text { MWd } \\
\text { was obtained on fuel elements } 164-0 \text { and } 164-\mathrm{I} \text {. Normal primary system } \\
\text { operating pressure was raised to } 750 \text { psig. The low pressure trip setpoint } \\
\text { was raised to } 600 \mathrm{psig} \text {, and the high inlet temperature trip setpoint was } \\
\text { lowered to } 130^{\circ} \mathrm{F} \text {. }\end{array}$ \\
\hline $3-26-77$ & 42.417 & $\begin{array}{l}\text { Fuel cycle } 144 \text { was terminated at } 4: 00 \mathrm{a} \cdot \mathrm{m} \text {. A total power generation of } 2345 \mathrm{MWd} \\
\text { was obtained on fuel elements } 140-0 \text { and } 140-\mathrm{I} \text {. The shutdown was extended to } \\
\text { complete the semiannual component inspection and to change the control plates. }\end{array}$ \\
\hline \multicolumn{3}{|r|}{ Unscheduled } \\
\hline $3-27-77$ & 0.133 & $\begin{array}{l}\text { The reactor scrammed at } 10 \mathrm{MW} \text { power level during startup due to high inlet } \\
\text { temperature from lack of secondary coolant flow. }\end{array}$ \\
\hline
\end{tabular}


PLANT MAINTENANCE

Maintenance and changes in the various.process systems are listed in Table 6.

\section{INSTRUMENTATION AND CONTROLS}

Maintenance and changes in the various instrumentation systems are listed in Table 7. 
Table 6. Process System - Maintenance and Changes

\begin{tabular}{|c|c|c|}
\hline Date & Component & Remarks \\
\hline & & Primary System \\
\hline $1-2-77$ & Pressurizer pump & $\begin{array}{l}\text { The magnetic clutch on pressurizer pump PU-4B was replaced because of } \\
\text { bearing failure. }\end{array}$ \\
\hline $3-7-77$ & Pressurizer pump & $\begin{array}{l}\text { The magnetic clutch on pressurizer pump PU-4B was replaced, again, } \\
\text { because of bearing failure. }\end{array}$ \\
\hline $3-25-77$ & Heat exchanger & $\begin{array}{l}\text { A leak of } \sim 0.1 \mathrm{gpm} \text { developed in the Cell } 110 \text { heat exchanger. The defec- } \\
\text { tive tube was located and plugs welded in both ends. }\end{array}$ \\
\hline $3-28-77$ & Heat exchanger & $\begin{array}{l}\text { A leak of } \sim 5 \mathrm{gpm} \text { developed in the Cell } 111 \text { heat exchanger. The defective } \\
\text { tube was located and plugs welded in both ends. }\end{array}$ \\
\hline \multirow[t]{2}{*}{$3-29-77$} & Pressurizer pump & $\begin{array}{l}\text { The suction seal on pressurizer pump } \mathrm{PU}-4 \mathrm{~B} \text { was replaced because of } \\
\text { excessive leakage. }\end{array}$ \\
\hline & & Miscellaneous \\
\hline $1-10-77$ & Acid pump & $\begin{array}{l}\text { The sulfuric acid pump motor was replaced after acid leaked through a } \\
\text { faulty valve onto the motor causing its windings to short out. }\end{array}$ \\
\hline $1-11-77$ & Air compressor & $\begin{array}{l}\text { The motor on instrument air compressor } C-1 B \text { was overhauled with new } \\
\text { bearings. }\end{array}$ \\
\hline
\end{tabular}


Table 7. Instrumentation - Maintenance and Changes

\begin{tabular}{llc}
\hline Date & Component & Remarks \\
\hline & Safety System \\
1-3-77 Signal converter & $\begin{array}{r}\text { The dual signal converter for safety chan- } \\
\text { nel No. } 3 \text { flux recorder failed. It was } \\
\end{array}$ & replaced with a spare. \\
\hline
\end{tabular}

\section{SYSTEM SURVEILLANCE TESTS AND RESULTS}

Vesse1 Head Studs

The accumulated number of tensioning cycles on the reactor vessel head studs is presented in Table 8 . These studs were designed for a fatigue life of 40 cycles loading due to tensioning of the bolts and $730 \mathrm{fu} 11-$ pressure ( $1000 \mathrm{psig}$ ) cycles. Installation of new reactor vessel head studs was completed in June, 1972. The numbers in Table 8 represent the maximum cycles to which any new stud has been exposed.

'lable 8. Vessel Head Stud-Tensioning Cycles

\begin{tabular}{lccc}
\hline I'ype Cycle & $\begin{array}{c}\text { This } \\
\text { Quarter }\end{array}$ & $\begin{array}{c}\text { Last } \\
\text { Quarter }\end{array}$ & $\begin{array}{c}\text { Toral } \\
\text { To Date }\end{array}$ \\
\hline Head bolts tensinner & 0 & 0 & 6 \\
1500 psig & & & 0 \\
950 psig & 0 & 0 & 7 \\
750 psig & 2 & 0 & 2 \\
650 psig & 3 & 3 & 117 \\
\hline
\end{tabular}

Semiannual Reactor-Component Inspection

At the end of fuel cycle 144, the semiannual reactor-component inspection was made. The shroud flange, fuel grid, and control plates were 
removed from the vessel. The components which remained in the vessel-upper and lower tracks, beryllium, beam tubes, and support pedestals--were inspected. No component abnormalities were detected.

\section{Stack Filters}

Stack filtering systems in the special building hot exhaust (SBHE) and hot off-gas (HOG) systems were tested for iodine removal efficiency. Results of the most recent tests are tabulated in Table 9. 
Table 9. Particulate and Iodine Removal Efficlency

\begin{tabular}{|c|c|c|c|c|c|c|c|c|c|c|c|c|c|}
\hline \multirow[b]{3}{*}{ F1lter Bank } & \multicolumn{4}{|c|}{ Methy I Iodice } & \multicolumn{4}{|c|}{ Elemental Iodine } & \multirow{3}{*}{$\begin{array}{c}\text { Filter } \\
\text { Position } \\
\end{array}$} & \multicolumn{4}{|c|}{ Particulate Retention } \\
\hline & \multicolumn{2}{|c|}{ Last Test } & \multicolumn{2}{|c|}{ Previous Test } & \multicolumn{2}{|c|}{ Last Test } & \multicolumn{2}{|c|}{ Previous Test } & & \multicolumn{2}{|c|}{ Last Test } & \multicolumn{2}{|c|}{ Previous Test } \\
\hline & Date & EFE., \% & Date & Eff., \% & Dace & Eff., $\%$ & Date & Eff., \% & & Date & Eff., $\%$ & Date & Eff., $\%$ \\
\hline \multirow[t]{2}{*}{ SBHE, west } & $11-9-76$ & $82 . \varepsilon i$ & $5-4-? 6$ & 48 & $10-8-7 \epsilon$ & 99.94 & $3-18-75$ & 99.96 & Soutí & $9-22-76$ & 99.993 & $3-26-76$ & 99.980 \\
\hline & & & & & & & & .. & Nort? & $9-22-76$ & 99.996 & $3-26-76$ & $99.000 *$ \\
\hline \multirow[t]{2}{*}{ SBHE, center } & $11-17-76$ & 87.14 & $5-11-76$ & 80 & $10-21-76$ & 90.98 & $3-30-76$ & 99.98 & Sout.z & $\mathrm{G}-22-76$ & 99.992 & $3-26-76$ & 99.9 .38 \\
\hline & & & & & & & & & Nort? & $9-22-76$ & 99.972 & $3-26-76$ & 99.960 \\
\hline \multirow[t]{2}{*}{ SBHE, east } & $11-12-76$ & 48.24 & $5-6-76$ & 47 & $10-14-76$ & 95.93 & $3-23-76$ & 99.97 & Saltị & $\subseteq-22-76$ & 99.971 & $3-26-76$ & 99.956 \\
\hline & & & & & & & & & North & $s-22-76$ & 99.992 & $3-26-76$ & $99.930 *$ \\
\hline HOG, west & $11-16-76$ & 99.17 & $5-13-76$ & 73 & $10-26-76$ & 99.99 & $4-6-76$ & 99.99 & $i$ & & & & \\
\hline HOG, center & $11-18-76$ & $98.5 \overline{5}$ & $5-18-76$ & 58 & $11-4-76$ & 99.99 & $4-22-7 \epsilon$ & 99.99 & . & & & & \\
\hline HOG, east & $11-10-76$ & 92.22 & $5-20-76$ & 98 & $10-12-75$ & 99.96 & $4-14-7 \epsilon$ & 99.90 & 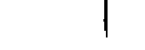 & & & & \\
\hline
\end{tabular}


REACTOR DESIGN CHANGES

Instrumentation and controls design change memoranda which describe work completed this quarter are tabulated in Table 10 .

Table 10. Instrumentation Change Memoranda

\begin{tabular}{|c|c|c|c|}
\hline $\begin{array}{l}\text { Memo } \\
\text { No. }\end{array}$ & Subject & \multicolumn{2}{|l|}{ Genera1 Description } \\
\hline \multirow[t]{12}{*}{89} & \multirow[t]{12}{*}{$\begin{array}{l}\text { Technical specifi- } \\
\text { cation conformance }\end{array}$} & \multicolumn{2}{|c|}{$\begin{array}{l}\text { To assure conformance with the technical } \\
\text { specifications for the HFIR, the set- } \\
\text { points for the indicated safety and con- } \\
\text { trol switches will be adjusted as } \\
\text { follows: }\end{array}$} \\
\hline & & a. Primary System Pressure & \\
\hline & & Operating pressure & $750 \mathrm{psig}$ \\
\hline & & Scram setpoint & 600 psig \\
\hline & & Primary pump cutoff & 400 psig \\
\hline & & Block valve closure & 675 psig \\
\hline & & $\begin{array}{l}\text { Start-standby } \\
\text { pressurizer pump }\end{array}$ & 700 psig \\
\hline & & Low pressure alarm & 700 psig \\
\hline & & High pressure alarm & 800 psig \\
\hline & & Pressurizer pump cutoff & 850 psig \\
\hline & & b. Reactor Inlet Temperature & \\
\hline & & Scram & $130^{\circ} \mathrm{F}$ \\
\hline
\end{tabular}

Mechanical design change memoranda which describe work completed this quarter are tabulated in Table 11.

Table 11. Mechanical Change Memoranda

Memo

No. Subject

General Description

52 Control plate bracket attachment screws
To increase the strength of the control plate bracket-to-the-control-plate attaching mechanism, stainless steel screws will be used on all remotely replaced brackets. 
REVTSTONS TO THE HFIR OPERATING MANUAL

Revisions and additions to the HFIR operating manual which have been published this quarter are tabulated in Table 12.

Table 12. HFIR Manual Revisions

\begin{tabular}{|c|c|c|c|}
\hline Date & $\begin{array}{c}\text { Section } \\
\text { No. } \\
\end{array}$ & Subject & Genera1 Description \\
\hline $3-22-77$ & $\begin{array}{l}\text { II-2.1 thru } \\
3.1 f\end{array}$ & $\begin{array}{l}\text { Technical speci- } \\
\text { fication } \\
\text { compliance }\end{array}$ & $\begin{array}{l}\text { Miscellaneous additions and up- } \\
\text { dating to conform with the } \\
\text { HFIR technical specifications } \\
\text { and setpoint ehänges. }\end{array}$ \\
\hline
\end{tabular}

\section{REACTOR EXPERTMENTS}

Experimental Facilities

Usage of the various HFIR experiment facilities is tabulated in Table 13.

Tablo 13. Exporimont Facility Uaage

\begin{tabular}{lll}
\hline Facility & \multicolumn{1}{c}{ Description } & \multicolumn{1}{c}{ Division } \\
\hline PTP-A1 & Materials studies & Metals and Ceramics \\
PTP-D1 & Materials studies & Metals and Ceramics \\
FIH-D7 & Materials studies & Metals and Ceramics \\
PTP-G4 & Materials studies & Metals and Ceramics \\
PTP-G7 & Materials studies & Metals and Ceramics \\
RB-1 & Isotope production & Operations \\
RB-2 & Ieotope production & Operations \\
RB-3 & Isotope production & Operations \\
RB-4 & HFIR tensile specimens & Operations \\
RB-5 & HTGR fuel irradiations & Engineering Technology \\
RB-6 & Isotope production & Operations \\
RB-7 & HTGR fuel irradiations & Engineering Technology \\
RB-8 & Isotope production & Operations
\end{tabular}


Table 13. (continued)

\begin{tabular}{lll}
\hline Facility & \multicolumn{1}{c}{ Description } & \multicolumn{1}{c}{ Division } \\
\hline CR-1 & Isotope production & Operations \\
CR-2 & Isotope production & Operations \\
CR-3 & Californium production & Chemical Technology \\
CR-4 & Californium production & Chemical Technology \\
CR-5 & Isotope production & Operations \\
CR-6 & Isotope production & Operations \\
CR-7 & Isotope production & Operations \\
CR-8 & Isotope production & Operations \\
VXF-1 & HFIR corrosion specimen & Operations \\
VXF-7 & Pneumatic tube & Analytical Chemistry \\
VXF-13 & HTGR fuel irradiations & Engineering Technology \\
EF-2 & Noise analysis studies & Instrumentation and Controls \\
& & and Operations \\
HB-1 & Neutron diffractometer & Solid State \\
HB-2 & Neutron diffractometer & Chemistry \\
HB-3 & Neutron diffractometer & Solid State \\
HB-4 & Neutron diffractometer & Solid State \\
\hline
\end{tabular}

HFIR Target Loading

A description of the HFIR target loading for each of the operating cycles this quarter is presented in Figures $1,2,3,4$, and 5. 


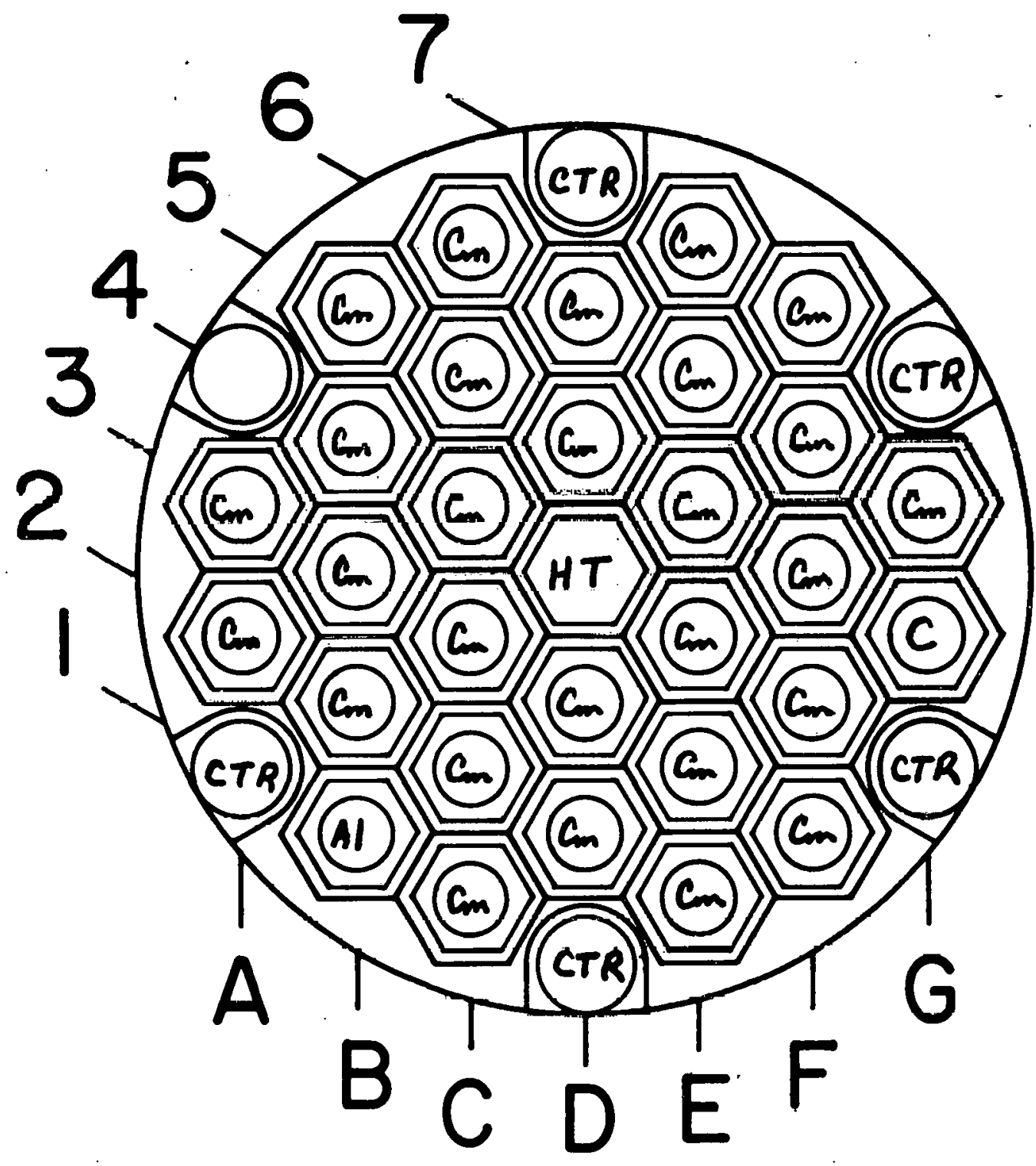

TARGET TYPE

NUMBER

PLUTONIUM (Pu)

CURIUM (Cm)

STAINLESS STEEL (SST)

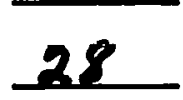

GRAPHITE (C)

ALUMINUM (AI)

HYDRAULIC TUBE (HT)

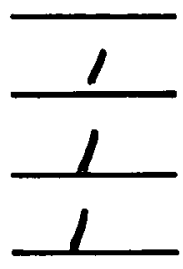

Fig. 1. HFIR Target Loading for Cycle 141 - December 15, 1976 


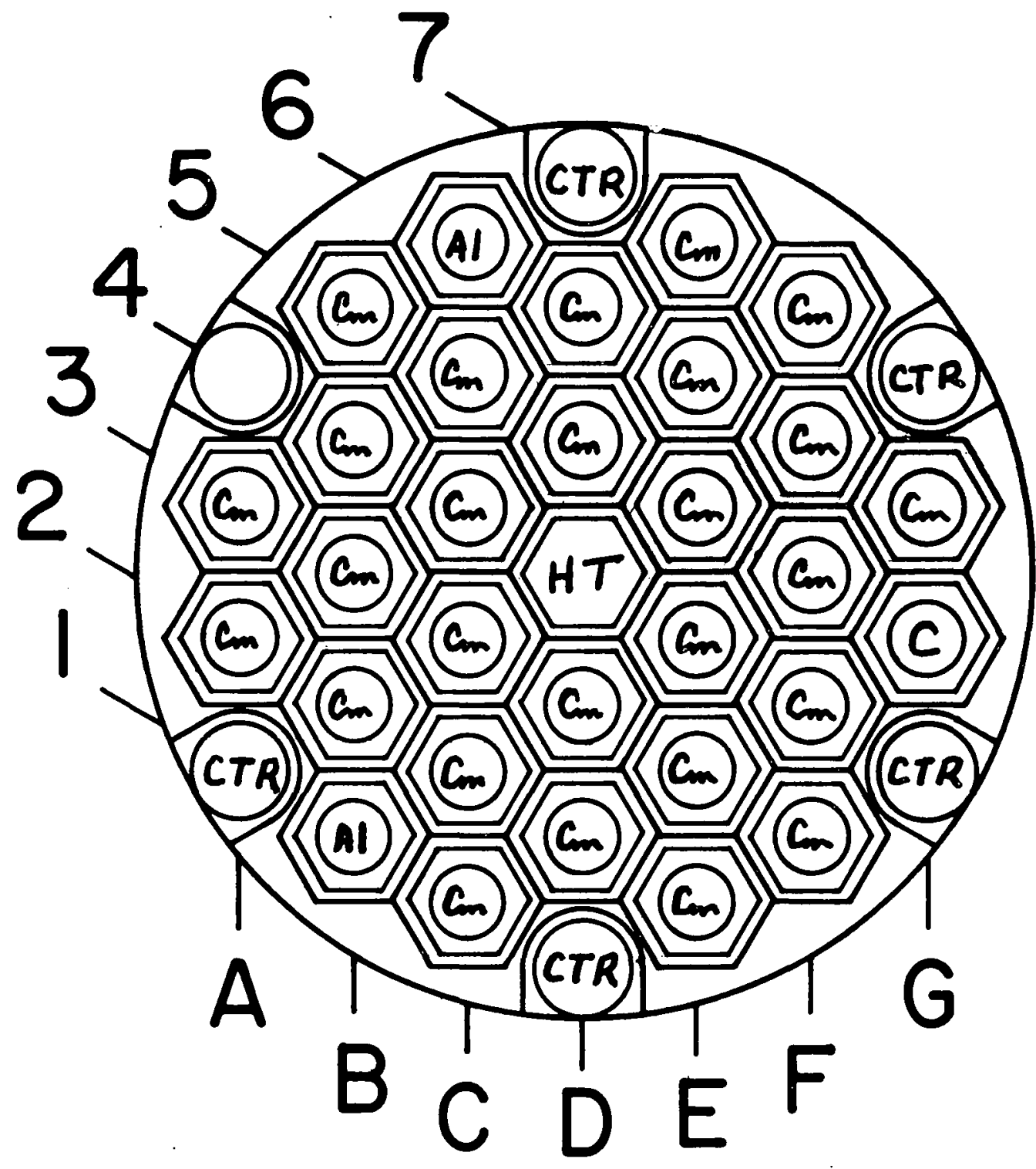

TARGET TYPE

NUMBER

PLUTONIUM (Pu)

CURIUM (Cm)

STAINLESS STEEL (SST)

GRAPHITE (C)

ALUMINUM (AI)

HYDRAULIC TUBE (HT)

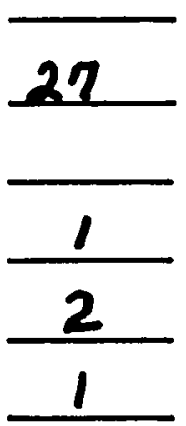

Fig. 2. HFIR Target Loading for Cycle 142 - January 10, 1977 


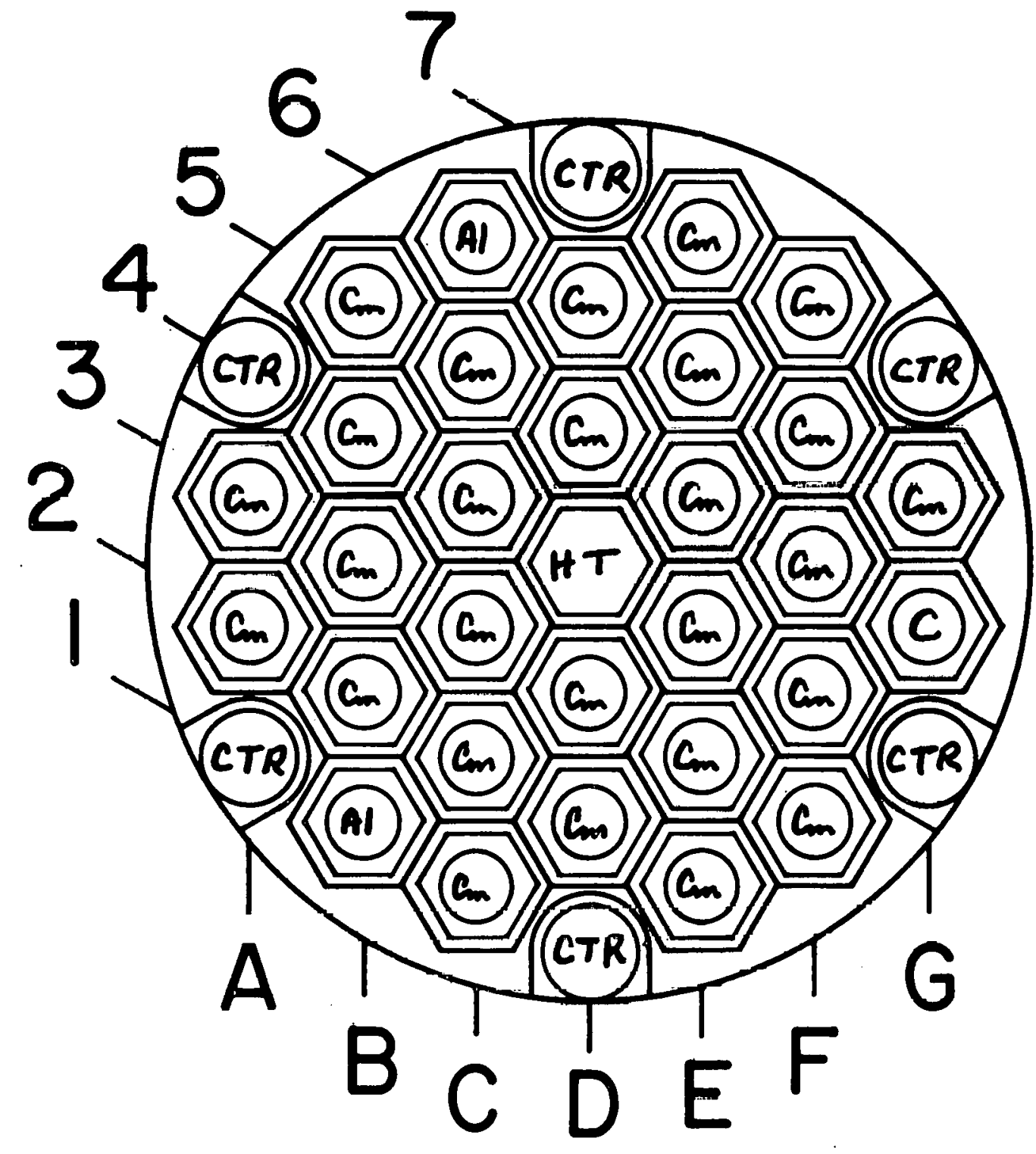

TARGET TYPE

NUMBER

PLUTONIUM (PU)

CURIUM (Cm)

STAINLESS STEEL (SST)

GRAPHITE (C)

ALUMINUM (AI)

HYDRAULIC TUBE (HT)

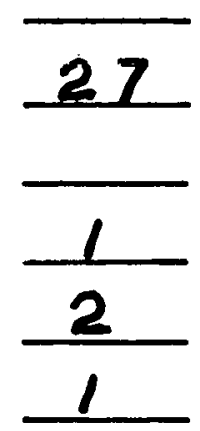

Fig. 3. HFIR Target Loading for Cycle 143 - February 5, 1977 


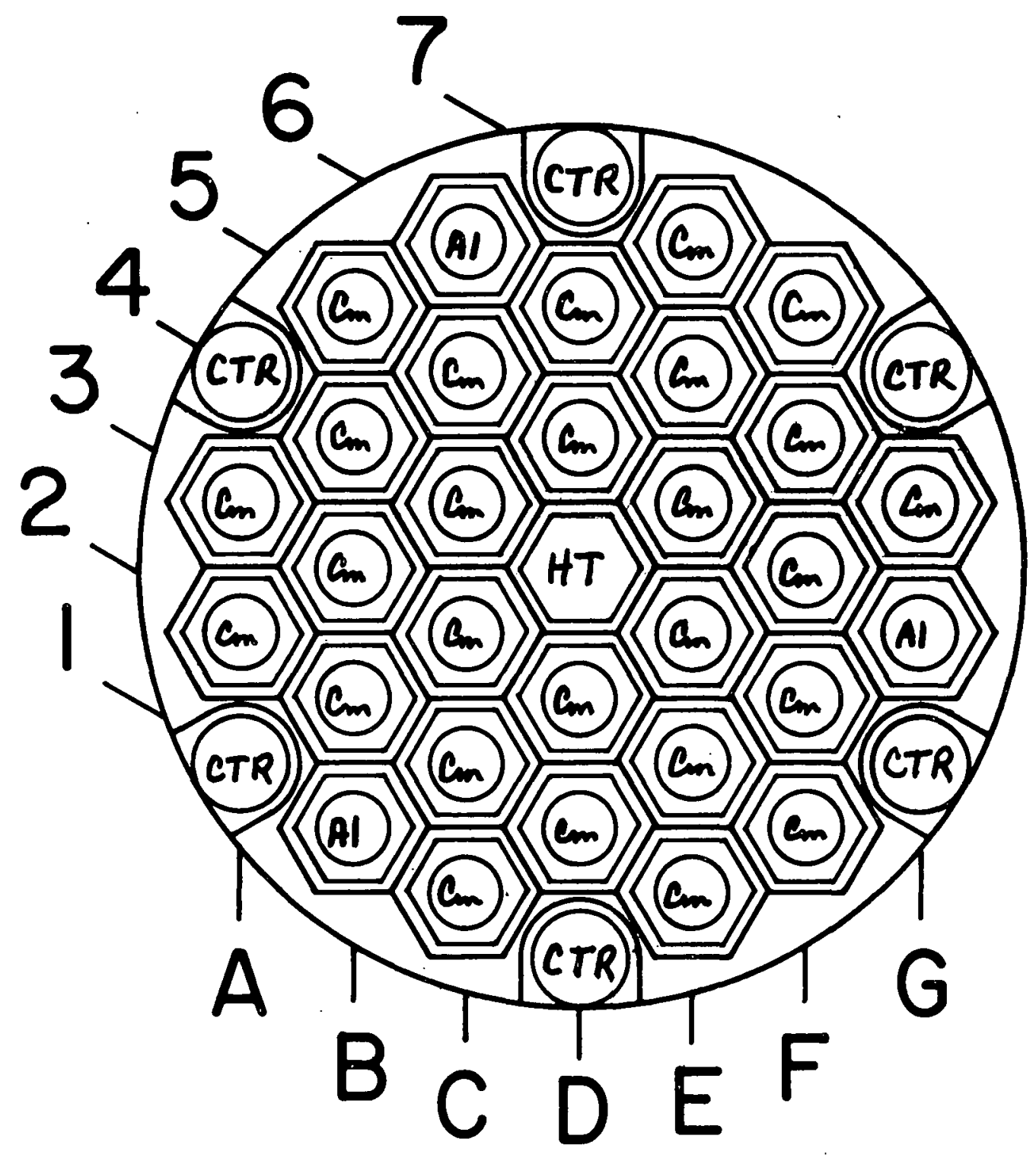

TARGET TYPE

NUMBER

PLUTONIUM (Pu)

CURIUM (Cm)

STAINLESS STEEL (SST)

GRAPHITE (C)

ALUMINUM (AI)

HYDRAULIC TUBE (HT)

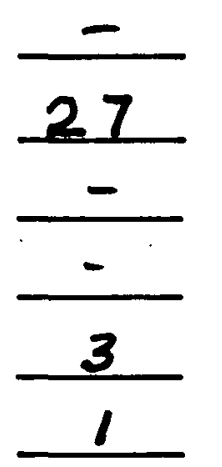

Fig. 4. HFIR Target Loading for Cycle 144 - March 2, 1977 


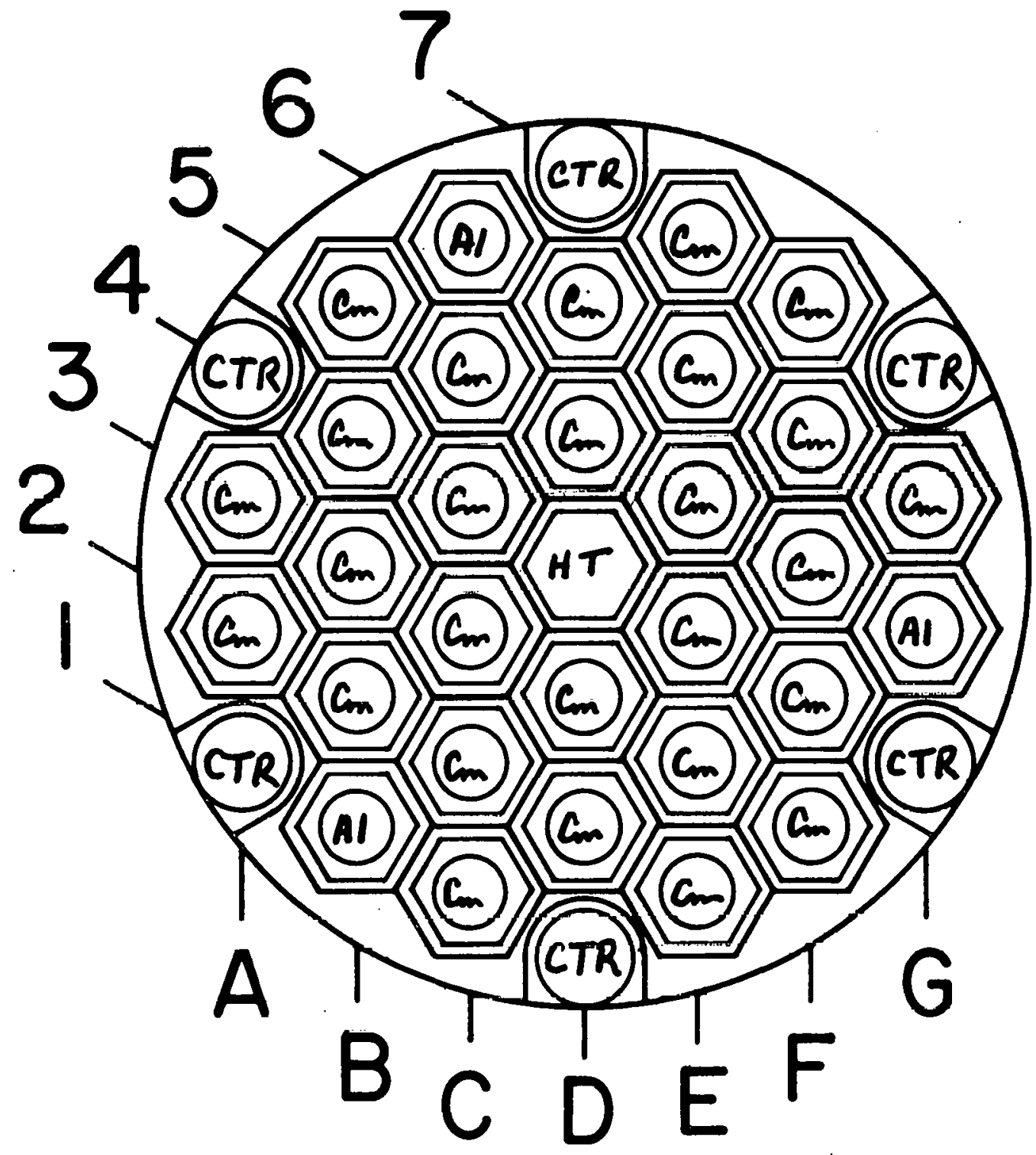

TARGET TYPE

NUMBER

PLUTONIUM (PU)

CURIUM (Cm)

STAINLESS STEEL (SST)

GRAPHITE (C)

ALUMINUM (AI)

HYDRAULIC TUBE (HT)

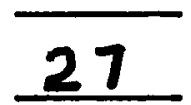

Fig. 5. HFIR Target Loading for Cycle 145 - March 27, 1977 
INTERNAL DISTRIBUTION

1. G. M. Adamson

2. J. L. Anderson

3. D. S. Asquith

4. K. S. Belitz

5. J. E. Bigelow

6. D. S. Billington

7. F. T. Binford

8. G. H. Burger

9. C. D. Cagle

10. W. R. Casto

11. R. D. Cheverton

12. T. E. Cole

13. J. A. Conlin

14-15. B. L. Corbett

16. J. A. Cox

17. W. H. Culbert

18. F. L. Culler

19. S. J. Ditto

20. G. J. Dixon

21. K. Farre11

22. E. D. Gupton

23. T. P. Hamrick

24. C. H. Helton

25. S. S. Hurt, III

26. E. B. Johnson

27. S. I. Kaplan

28. 0. L. Keller

29. E. M. King

30. H. V. Klaus

31. R. W. Knight
32. W. C. Koehler

33. E. Lamb

34. H. A. Levy

35. J. Lewin

36-41. R. V. McCord

42. J. R. McGuffey

43. H. A. McLain

44. J. R. McWherter

45. J. M. Miller

46. L. C. Oakes

47. H. Postma

48. K. H. Poteet

49. M. E. Ramsey

50. R. L. Scott, Jr.

51. W. H. Sides

52. T. M. Sims

53. M. J. Skinner

54. J. H. Swanks

55. J. R. Thomas

56. D. B. Trauger

57. J. R. Weir

58. K. W. West

59. F. W. Wiffen

60. M. K. Wilkinson

61. J. W. Woods

62-63. Central Research Library

64. Document Reference Section

65-66. Laboratory Records Department

67. Laboratory Records, ORNL R.C.

\section{EXTERNAL DISTRIBUTION}

68-94. Technical Information Center, Oak Ridge

95-96. M. B. Biles, Director, Division of Safety, Standards, and Compliance, ERDA, Washington, D. C. 20545

97. K. J. Bobin, Centre D'Etude de Energie Nucleaire, S.C.K./C.E.N, Boeretang 200, B-2400 Mol, Belgium

98. J. L. Burnett, Division of Physical Research, ERDA, Washington, D. C. 20545

99. H. N. Culver, ERDA, Oak Ridge

100. M. Jacqucmain, Head, Technical Department ILL, Institute Max von Laue-Paul Langevin, CEDEX 156, 38 Grenoble-Gare, France

101. C. L. Rogosa, Division of Physical Research, ERDA, Washington, D. C. 20545

102. Research and Technical Support Division, ERDA, Oak Ridge 